\title{
Biological activities of pargamicin A, a novel cyclic peptide antibiotic from Amycolatopsis sp.
}

Hideki Hashizume, Hayamitsu Adachi, Masayuki Igarashi, Yoshio Nishimura and Yuzuru Akamatsu

The Journal of Antibiotics (2010) 63, 339-340; doi:10.1038/ja.2010.37

Correction to: The Journal of Antibiotics (2010) 63, 279-283; doi:10.1038/ja.2010.29 wrong symbol was used to denote the absence of drug concentrations. The corrected figures and captions are shown below.

The authors of the above article noted an error in publication of this paper (AOP and in this issue) in Figures 4 and 5, whereby the

a

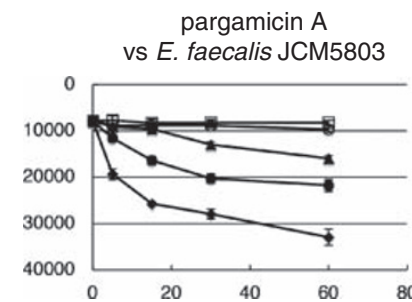

b

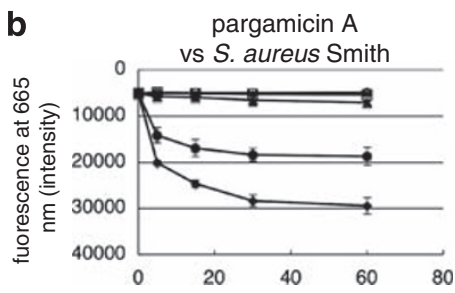

C

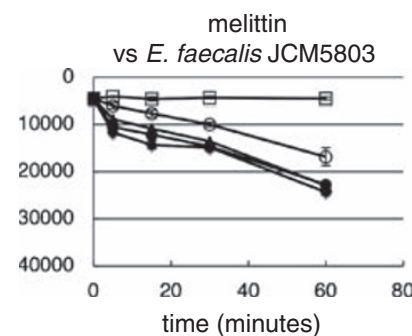

d

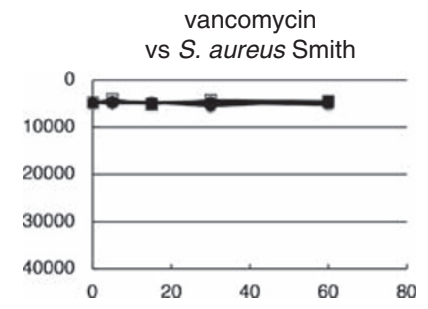

e

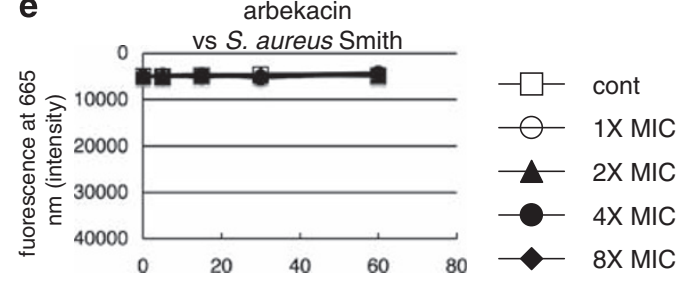

f



Figure 4 Effect of pargamicin A (a, b), melittin (c), vancomycin (d), arbekacin (e) and daptomycin (f) on membrane potential. Each point is the mean \pm s.d. of four estimates. Membrane potential assays were evaluated in the absence (open squares) or presence of the following concentrations of drugs: $1 \times$ MIC (open circles), $2 \times$ MIC (solid triangles), $4 \times$ MIC (solid circles) and $8 \times$ MIC (solid diamonds). 
a

pargamicin $A$ vs E. faecalis JCM5803

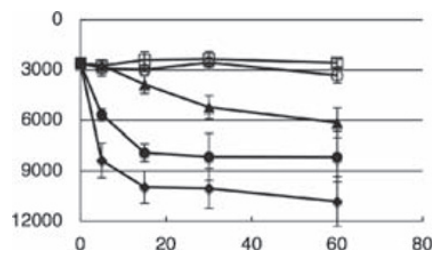

b
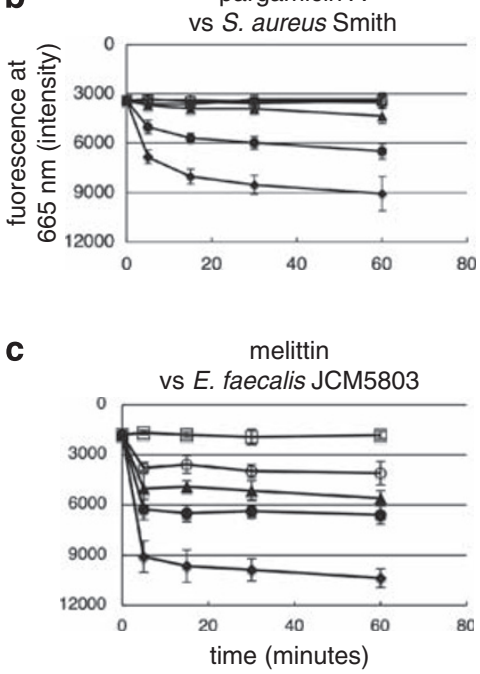

d

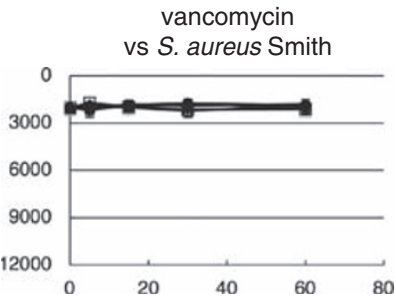

arbekacin

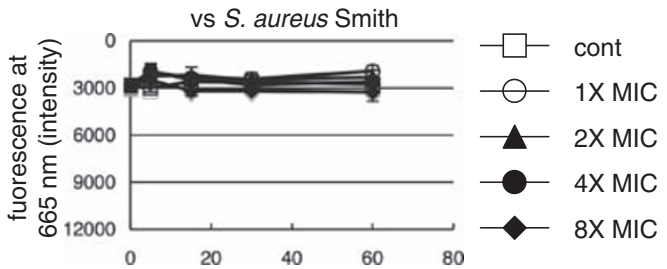

f

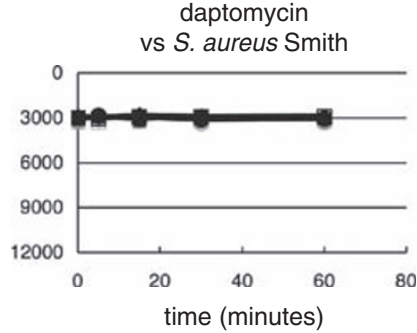

Figure 5 Loss of membrane function by pargamicin A (a, b), melittin (c), vancomycin (d), arbekacin (e) and daptomycin (f). Each point is the mean \pm s.d. of four estimates. Membrane function assays were evaluated in the absence (open squares) or presence of the following concentrations of drugs: $1 \times$ MIC (open circles), $2 \times$ MIC (solid triangles), $4 \times$ MIC (solid circles) and $8 \times$ MIC (solid diamonds). 